\title{
Late termination of pregnancy for fetal anomalies: Experience at a tertiary-care hospital in South Africa
}

\author{
F Shahid, MBBS, FCOG (SA), Cert Maternal and Fetal Medicine (SA); \\ P Soma-Pillay, MB ChB, Dip Obstet (SA), FCOG (SA), Cert Maternal and Fetal Medicine (SA), PhD
}

Department of Obstetrics and Gynaecology, Faculty of Health Sciences, University of Pretoria and Steve Biko Academic Hospital, Pretoria, South Africa

Corresponding author: F Shahid (Fauziashams25@gmail.com)

\begin{abstract}
Background. Early diagnosis and appropriate management of congenital anomalies can help prevent neonatal morbidity and mortality. Termination of pregnancy for severe congenital anomalies is permitted under South African law.

Objective. To determine factors causing delayed diagnosis of lethal congenital abnormalities requiring late termination of pregnancy at Steve Biko Academic Hospital in Pretoria, South Africa.

Methods. Medical records of pregnant women who presented with lethal fetal anomalies over a period of 7.5 years were analysed. Patients' demographic profile, the interval from referral to feticide, gestational age at first scan and diagnosis, type of anomaly and feticide methods were considered. The cohort was divided in two groups based on timing of termination (i.e. earlier than 28 weeks and later than 28 weeks' gestation) for statistical comparison.

Results. The majority of women $(n=45 ; 78.9 \%)$ were younger than 35 years and had no chronic medical conditions or risk factors $(n=40$; $70 \%)$. Although 30 women (52.6\%) had been booked for antenatal examination early in their pregnancy, only three (5.2\%) had a firsttrimester scan. Mean time to referral was not significantly different between the women whose pregnancies terminated earlier than 28 weeks and those with a termination after 28 weeks $(p=0.671)$.

Conclusion. A basic ultrasound scan in the second trimester is recommended for all pregnant women. Task shifting can be a viable option to provide this facility at primary and secondary health centres. A national registry should be established to document all late terminations for fetal anomalies.
\end{abstract}

S Afr J Obstet Gynaecol 2019;25(2):35-40. https://doi.org/10.7196/SAJOG.2019.v25i2.1402

Severe congenital anomalies are reported in $2 \%$ of births and cause frequent hospitalisations and disabilities. ${ }^{[1]}$ Permanent disability of a child has considerable psychological and financial implications for the family and also places demands on healthcare systems. Despite major advances in prenatal screening for major congenital anomalies over the past few decades, therapeutic options remain limited. ${ }^{[1,2]}$ With technological advances and more accessible and affordable healthcare, many severe abnormalities are now diagnosed early in pregnancy, specifically in high-income countries. ${ }^{[1]}$ In these countries, various policies and recommendations have been developed to avoid missing a major abnormality until late in gestation. ${ }^{[1]}$

The situation in low-to-middle-income countries is somewhat different. Basic cost of travel, women's difficulty in accessing antenatal healthcare or late booking, inadequate training of healthcare workers and late appearance of certain anomalies are the main reasons for delayed diagnosis. ${ }^{[3]}$

These factors have contributed to the decision by many countries to allow parents to request a late termination of pregnancy (LTOP) for fetal anomalies. ${ }^{[2]}$ Abortion laws differ from country to country and, owing to ethical reasons, issues become more complex as the pregnancy advances into the third trimester ${ }^{[4]}$ In most countries, moral status is conferred on the fetus after 24 weeks of gestation, but it varies from post viability (e.g. in the USA) to post delivery (e.g. in Israel and South Africa (SA)). ${ }^{[4]}$ Under the Choice of Termination of Pregnancy Act No. 92 of 1996, termination is legal in SA and under specific conditions there is no upper limit for performing a termination. At a woman's request, a physician or a trained midwife can perform a termination during the first 12 weeks of gestation. From the thirteenth to the twentieth week of gestation, a physician may, in consultation with the mother, terminate the pregnancy if it presents a threat to the mother's physical or mental health or in circumstances related to socioeconomic constraints. Only the pregnant woman's consent is required. After the twentieth week of gestation, a termination may be performed if a medical practitioner, in consultation with another authoritative healthcare worker, is of the opinion that continued pregnancy will endanger the woman's life or health or if there is a substantial risk of severe physical or mental abnormalities if the child were born. ${ }^{[5]}$

LTOPs, defined as terminations after 24 weeks of gestation, should be performed only after a multidisciplinary team's assessment of the situation. ${ }^{[3]}$ Women should be given a choice of active or expectant management and be informed that, in special circumstances, feticide is ethically justified to ensure that the fetus is stillborn. ${ }^{[5,6]}$

There is a paucity of data regarding LTOP in SA, its indications and the prevalence of specific congenital anomalies. Feticide is performed at many tertiary institutions, yet there is no national registry to record the number of procedures or the technique used. ${ }^{[3]}$

Techniques described for feticide, either in singleton or multiple pregnancies, include fetal intracardiac, -umbilical cord or -cerebral injection of potassium chloride $(\mathrm{KCl})$, lignocaine or a combination 
of these agents, or intrapericardial instillation of normal saline to cause a cardiac tamponade. Whichever method is used, it should be effective and safe for the mother. ${ }^{[3]}$

The aim of our study was to determine the indications for feticide, the technique used and the reasons for late referral into the healthcare system.

\section{Methods}

This was a retrospective study at the Maternal and Fetal Medicine Unit of the Steve Biko Academic Hospital (SBAH) in Pretoria, SA, from 1 January 2010 to 1 July 2017. The unit is a tertiary referral centre for the diagnosis and management of fetal abnormalities. Medical records of all women whose pregnancies (singleton or multiple) were terminated after 24 weeks owing to fetal anomalies were included for analysis. The study population was subsequently divided into two groups, namely terminations between 24 and 28 weeks of gestation and terminations after 28 weeks of gestation at the time of feticide. Permission to perform this audit was obtained from SBAH and the Faculty of Health Sciences' Research Ethics Committee at the University of Pretoria.

\section{Late termination of pregnancy}

The decision to offer LTOP was made by two consultants working in the unit. If indicated, the services of other specialists (e.g. a paediatric cardiologist, radiologist, geneticist or social worker) were offered to the patient. Women at high risk for fetal anomalies and still in their first trimester were referred from primary and secondary hospitals and private institutions for aneuploidy screening. If indicated, invasive testing was offered. Women in their second trimester were generally referred for a detailed anatomy scan using an EPIQ 5 ultrasound scanner, with C5/1 and C103V probes (Philips, The Netherlands) or a Voluson S-6 system, with 25 RS and 5/9WRS probes (GE Healthcare, Norway). We used two- and threedimensional scanning, as well as pulsed-wave Doppler and colour Doppler scanning functions, as was indicated.

A consent form was signed after the decision to terminate the pregnancy. Feticide was performed by injecting either $\mathrm{KCl}$ or a combination of lignocaine and $\mathrm{KCl}$ into the fetal heart until asystole was observed. Feticide was deemed successful if no fetal cardiac activity was observed after the procedure. After feticide, the mother was admitted for induction of labour or transferred to her referring hospital.

\section{Statistics}

Descriptive parameters were expressed as means and standard deviations for normally distributed data. Data not distributed normally were expressed as medians and ranges. Independentsamples $t$-tests were used to compare normally distributed data, whereas the Mann-Whitney $U$ test was used for data not distributed normally. A significance level of $p<0.05$ was used.

\section{Results}

A total of 23320 deliveries were recorded at SBAH between 1 January 2010 and 1 July 2017, of which 1318 were stillbirths. During this period, 100 feticide procedures were performed. Only women who were to have the feticide procedure and delivery performed at SBAH were included in study $(n=57)$. One of these women declined feticide. Data of women who had the feticide procedure performed at SBAH but were subsequently transferred back to the referring institution for delivery $(n=43)$ were excluded from the analysis.

\section{Demographic data}

Demographic data of the study population are shown in Table 1. The majority $(78.9 \%)$ of women who presented with structural fetal anomalies that justified feticide were younger than 35 years. A large proportion of the cohort $(70.1 \%)$ were considered to have a low risk of fetal anomalies. Of the women whose antenatal examination booking was later than at 24 weeks $(n=24)$, two $(8.3 \%)$ were employed and 15 (62.5\%) were unemployed; the employment status of the remainder $(29.2 \%)$ was unknown. In the group whose bookings were earlier than 24 weeks $(n=30)$, four (13.3\%) were employed and 17 (56.7\%) were unemployed; the remainder's (30\%) employment status was not known. Among the women whose records did not show an antenatal booking $(n=3)$, one was employed and another was unemployed; the employment status of the third woman was unknown.

Two women were diabetic. In both cases, the diagnosed fetal abnormalities affected the central nervous system although karyotypes were found to be normal.

The majority of the sample $(n=54 ; 94.7 \%)$ had no history of illicit drug use or treatment with warfarin or a teratogenic agent; in three cases (5.3\%) no records of possible exposure to teratogenic agents were available.

The systems affected are listed in Table 2. Amniocentesis had been performed in 40 cases $(70.2 \%)$; no invasive testing was performed in the remainder $(n=17 ; 29.8 \%)$. Only one of the four mothers with

\begin{tabular}{ll} 
Table 1. Demographics of the study population $(\boldsymbol{N}=57)$ \\
\hline Variables & $\boldsymbol{n}(\%)$ \\
\hline Age, years & $27(6.7)$ \\
Mean (SD) & $16-41$ \\
Range & $45(78.9)$ \\
$<35$ years & $12(21.1)$ \\
$>35$ years & \\
Race & $48(84)$ \\
Black & $8(14)$ \\
White & $1(2)$ \\
Coloured & \\
Marital status & $13(23)$ \\
Married & $38(67)$ \\
Single & $6(10.5)$ \\
Unknown & \\
Chronic medical conditions & $2(3.5)$ \\
Diabetes & $3(5.2)$ \\
Hypertension & $12(21)$ \\
HIV infection & $40(70.2)$ \\
None & $0(0)$ \\
Congenital abnormalities in family & \\
Antenatal booking status & $30(52.6)$ \\
Booked $<24$ weeks & $24(42.1)$ \\
Booked $>24$ weeks & $3(5.2)$ \\
No booking & \\
Parity status & $7(12.2)$ \\
3 & $10(17.5)$ \\
2 & $24(42.1)$ \\
1 & $16(28)$ \\
0 & \\
&
\end{tabular}


RESEARCH

Table 2. Description of fetal anomalies and affected systems seen in reviewed cases $(N=57)$

\begin{tabular}{|c|c|c|c|}
\hline Affected system & Predominant anomaly & Associated anomaly & Karyotype $^{*}$ \\
\hline & Dandy-Walker malformation & $\begin{array}{l}\text { Clubbed feet; clenched hands; } \\
\text { omphalocele; hypoplastic lungs }\end{array}$ & Trisomy 13 \\
\hline & Spina bifida; Dandy-Walker malformation & Hypoplastic left heart; omphalocele & Trisomy 18 \\
\hline & Hydrocephaly & & $46, \mathrm{XY}$ \\
\hline & Hydrocephaly & & $46, X Y$ \\
\hline & Alobar holoprosencephaly & & $46, \mathrm{XX}$ \\
\hline & Holoprosencephaly & & $46, \mathrm{XX}$ \\
\hline & Bilateral ventriculomegally & Multicystic kidney disease & $46, \mathrm{XX}$ \\
\hline & Alobar holoprosencephaly & & $46, \mathrm{XX}$ \\
\hline & Alobar holoprosencephaly & & $46, \mathrm{XX}$ \\
\hline & Hydrocephalus & & $46, X Y$ \\
\hline & Holoprosencephaly & Tetralogy of Fallot; clubbed feet & $46, \mathrm{XX}$ \\
\hline & Anencephaly & & $46, X Y$ \\
\hline & Hydrocephalus & & $46, X Y$ \\
\hline & Cerebellar aplasia & Skeletal dysplasia & $46, \mathrm{XX}$ \\
\hline & Cerebellar aplasia & Skeletal dysplasia (thanatophoric) & $46, \mathrm{XX}$ \\
\hline & Chiari malformation & & $46, X Y$ \\
\hline & Semilobar holoprosencephaly & & $46, \mathrm{XX}$ \\
\hline & Chiari malformation & & $46, X Y$ \\
\hline & Alobar holoprosencephaly & & $46, \mathrm{XX}$ \\
\hline & Hydrocephalus & & $46, X Y$ \\
\hline & Encephalocele & & $46, \mathrm{XX}$ \\
\hline \multirow[t]{23}{*}{ Central nervous system } & Encephalocele & & $46, X Y$ \\
\hline & Hydrocephalus & & $46, \mathrm{XX}$ \\
\hline & Holoprosencephaly & & Not done \\
\hline & Porencephalic cyst & & Not done \\
\hline & Holoprosencephaly & & Not done \\
\hline & Encephalocele & & Not done \\
\hline & Hydranencephaly & Clubbed feet & Not done \\
\hline & Holoprosencephaly & Ambiguous genitalia & Not done \\
\hline & Alobar holoprosencephaly & & Not done \\
\hline & Chiari malformation; spina bifida & & Not done \\
\hline & Hydrocephalus & & Not done \\
\hline & Holoprosencephaly & & Not done \\
\hline & Holoprosencephaly & & Not done \\
\hline & Holoprosencephaly & Spina bifida & Not done \\
\hline & Schizencephaly; hydrodrocephaly & & $\mathrm{n} / \mathrm{a}^{\dagger}$ \\
\hline & Chiari malformation; spina bifida & Skeletal dysplasia & $\mathrm{n} / \mathrm{a}$ \\
\hline & Vermian agenesis and corpus callosum agenesis & & $\mathrm{n} / \mathrm{a}$ \\
\hline & Hydrocephalus aqueductal stenosis & & $\mathrm{n} / \mathrm{a}$ \\
\hline & Chiari malformation & Spina bifida & $\mathrm{n} / \mathrm{a}$ \\
\hline & Intraventricular haemorrhage & & $\mathrm{n} / \mathrm{a}$ \\
\hline & Hydrocephalus & & $\mathrm{n} / \mathrm{a}$ \\
\hline & Dandy-Walker malformation & & $\mathrm{n} / \mathrm{a}$ \\
\hline & Hydrocephalus & & Not done \\
\hline \multirow[t]{2}{*}{ Cardiovascular system } & Hypoplastic left heart & & $46, \mathrm{XY}$ \\
\hline & Hypoplastic left heart & Bilateral pyelectasis; echogenic bowel & $46, \mathrm{XY}$ \\
\hline \multirow[t]{2}{*}{ Gastrointestinal system } & Duodenal atresia & Clenched hands; clubbed feet & Trisomy 18 \\
\hline & Duodenal atresia & Clenched hands & Trisomy 18 \\
\hline \multirow[t]{4}{*}{ Skeletal system } & Skeletal dysplasia & Macrocephaly & $46, \mathrm{XY}$ \\
\hline & Skeletal dysplasia & Transposition of great arteries & $46, \mathrm{XY}$ \\
\hline & Skeletal dysplasia & Agenesis of corpus callosum & $\mathrm{n} / \mathrm{a}$ \\
\hline & Skeletal dysplasia & Agenesis of corpus callosum & $\mathrm{n} / \mathrm{a}$ \\
\hline Renal system & Posterior urethral valves & & Not done \\
\hline Respiratory system & Hypoplastic lungs & Omphalocele & Failed \\
\hline \multirow[t]{3}{*}{ Other } & Parapagus twins & & Not done \\
\hline & Thoracopagus twins & & Not done \\
\hline & Hydrops fetalis due to parvovirus B19 infection & & Not done \\
\hline
\end{tabular}


aneuploid fetuses was older than 35 years. Three (of the 25) women with euploid fetuses were older than 35 years.

Two cases of conjoined twins were noted. One case was missed at the first-trimester ultrasound scan (14 weeks' gestation) and subsequently diagnosed only late in the second trimester. The second case occurred in a patient who booked for antenatal examination only after 24 weeks of gestation. The patient in whom hydrops fetalis was diagnosed was referred to our unit at 16 weeks of gestation and subsequently received two intrauterine blood transfusions; however, the fetal status did not improve. The patient opted for the feticide procedure after 25 weeks of gestation owing to a poor prognosis.

Severe central nervous system abnormalities were found in 44 cases (77.2\%). Abnormalities affecting multiple systems were seen in 19 cases (33.3\%). Hypoplastic left heart syndrome and posterior urethral valves as primary anomaly were seen in one case each, although hypoplastic left heart syndrome also occurred as an associated anomaly in two other cases.

Table 3 shows details of the ultrasound scan and feticide procedures. Eight women (14\%) had their first scan at SBAH, of

\begin{tabular}{ll} 
Table 3. Description of ultrasound scan and feticide \\
procedure \\
\hline Description & $\boldsymbol{n}(\%)$ \\
\hline Facility of first ultrasound scan $(N=57)$ & $23(40.3)$ \\
$\quad$ Private doctor & $20(35.1)$ \\
Secondary institution & $8(14.0)$ \\
Tertiary institution (SBAH) & $6(10.5)$ \\
No scan on referral & \\
Gestational age at first ultrasound $(N=57)$ & $3(5.3)$ \\
First trimester & $12(21.0)$ \\
Second trimester & $42(73.7)$ \\
Third trimester & \\
Feticide procedure $(N=56)$ & $39(69.6)$ \\
Intracardiac injection & $5(8.9)$ \\
Intraumbilical cord injection & $3(5.4)$ \\
Intracerebral injection & $9(16.1)$ \\
Unknown & \\
Agent used in feticide procedure $(N=56)$ & $35(62.5)$ \\
KCl & $0(0)$ \\
Lignocaine & $9(16)$ \\
KCl-lignocaine & $12(21)$ \\
Unknown & \\
Procedure outcome $(N=56)^{*}$ & $52(92.9)$ \\
Successful & $4(7.1)$ \\
Failed & \\
*One patient declined feticide. & \\
BBAH = Steve Biko Academic Hospital. & \\
&
\end{tabular}

whom six were referred for advanced maternal age and two for type 1 diabetes after examination at their local clinic.

Fetal asystole was achieved at the first attempt in 27 cases and at the second in 24 cases. The maximum number of attempts recorded to achieve fetal asystole was six. The dose of feticidal agent was not recorded in 24 cases. In the remainder, $5-15 \mathrm{~mL}$ of $10 \%(\mathrm{w} / \mathrm{v}) \mathrm{KCl}$ was used in 23 cases, while $<5 \mathrm{~mL}$ and $>15 \mathrm{~mL}$ was required in two and seven cases, respectively. In the case for which the maximum dose $(37 \mathrm{~mL})$ was recorded, the feticidal agent was a combination of $25 \mathrm{~mL} \mathrm{10 \% (w/v)} \mathrm{KCl}$ and $12 \mathrm{~mL} \mathrm{2 \% (w/v)} \mathrm{lignocaine.} \mathrm{No}$ complications due to the dose of $\mathrm{KCl}$ or its combination with lignocaine were reported.

In four cases, the heart or umbilical cord was not accessible and therefore the feticidal agent $(\mathrm{KCl})$ was injected intracerebrally. A procedure was declared failed if fetal asystole was not achieved and labour was subsequently induced. Stillborn fetuses were delivered in three such cases; in one case the fetus was born alive but demised shortly after birth.

Factors assessed in late and very late terminations (at $24-28$ weeks' and at $>28$ weeks' gestation, respectively) are compared in Table 4.

\section{Discussion}

As advanced maternal age is a risk factor for aneuploid conception, women older than 35 years are offered screening for fetal abnormalities in SA. ${ }^{[7]}$ However, our findings suggest that such ultrasound screening should be considered for all pregnant women, irrespective of their risk profile. SA maternity care guidelines recommend that every low-risk pregnant woman should, wherever resources allow, have a basic ultrasound scan at 18 - 20 weeks' gestation performed by an accredited sonographer to determine fetal viability, number of fetuses, basic gestational age, location of the placenta and to assess the volume of amniotic fluid. ${ }^{[7]}$ Owing to capacity constraints, sonographers are not readily available and midwives and family physicians can perform these scans at primarycare centres. ${ }^{[7]}$ Effectiveness of a prenatal screening programme depends on the type of defect, quality of equipment, the operator's experience and the country's law regarding termination of pregnancy. ${ }^{[8]}$ Data from the EUROCAT registry suggests that $64 \%$ of birth defects can be diagnosed prior to delivery, although detection rates vary from $94 \%$ for anencephaly and $87 \%$ for omphalocele to $27 \%$ for transposition of great arteries. ${ }^{[1]}$

Owing to financial constraints, developing countries face many challenges in providing high-quality antenatal care, including a lack of equipment and trained staff and low patient compliance. ${ }^{[2]}$ Such factors may have contributed to the late diagnosis of anomalies and consequent late terminations in our study.

Table 4. Aspects related to feticide decision and procedure in late and very late termination of pregnancy

\begin{tabular}{|c|c|c|c|}
\hline Variable & Late termination ${ }^{*}(N=9)$ & Very late termination ${ }^{*}(N=47)$ & $p$-value \\
\hline Maternal age (years), mean (SD) & $28.33(8.91)$ & $27.55(5.75)$ & 0.807 \\
\hline Gestational age at booking (weeks), mean (SD) & $18.63(3.89)^{\dagger}$ & $24.27(7.2)^{\ddagger}$ & 0.04 \\
\hline Time to referral (days), median (range) & $8(1-41)$ & $6.5(1-70)$ & 0.671 \\
\hline Time to feticide (days), median (range) & $28(1-70)$ & $4(1-105)$ & 0.168 \\
\hline Time to delivery (days), mean (SD) & $1.56(0.73)$ & $1.64(0.85)$ & 0.785 \\
\hline
\end{tabular}


Prenatal diagnosis of anomalies can decrease perinatal mortality if timely termination is performed or appropriate medical or surgical intervention can be identified. ${ }^{[8]}$ Poestoev et al. ${ }^{[8]}$ reported a fivefold decline in the perinatal mortality rate in neonates with lethal anomalies in Russia (from 106.6/1 000 to 21.2/1 000) after prenatal screening had been implemented, probably due to timely termination of pregnancy upon detection of severe fetal anomalies. However, results reported by the RADIUS study group in the USA did not support this finding. ${ }^{[9]}$ Abortion being illegal in certain states of the USA but allowed in Russia could have contributed to the difference.

All but one of the women in our cohort accepted LTOP. Acceptance of LTOP related to prenatal diagnosis of a severe fetal anomaly depends on various factors, including a country's laws, the mother's religious beliefs and the availability of services. ${ }^{[3,4]}$ In Europe, the prevalence of termination of pregnancy for fetal anomaly varies from 0/1 000 births in countries such as Ireland, Malta and Poland, where terminations after 24 weeks is illegal, to $10 / 1000$ in France, where LTOP is allowed. Acceptance of LTOP due to fetal anomaly ranges from $15 \%$ to $59 \%$ in Europe. ${ }^{[1]}$

Early booking for antenatal care allows early diagnosis of abnormalities and appropriate management. Studies have shown that women in low-income countries perceive ultrasound scanning as a positive intervention, probably as the pregnancy is associated with low risk in the majority of cases. ${ }^{[10]}$ When used as an adjunct to antenatal care at local clinics, ultrasound scanning has been shown to improve antenatal clinic attendance and referral to a highercare facility for delivery. ${ }^{[10]}$ In the context of low-to-middle-income countries, more regular attendance also allows for services such as HIV testing, treatment of anaemia, deworming, blood pressure testing and education on nutrition and neonatal care. ${ }^{[10]}$

In low-resource settings, implementation of low-cost ultrasound programmes focused on identifying high-risk conditions of pregnancy and appropriate triage and referral can have a beneficial effect on perinatal morbidity and mortality. ${ }^{[10]}$ However, potential benefits should be weighed against the burden on available facilities, the risk of overtreatment due to false positives and possible detraction from other beneficial services. ${ }^{[10]}$ Use of relatively inexpensive ultrasound equipment and lowering the running costs (e.g. by using olive oil as a lubricant and not printing photos) are feasible options for implementing low-cost programmes. ${ }^{[10]}$

Provision of skilled personnel incurs the greatest cost and can be addressed by task shifting and training midwives and nurses to perform basic ultrasound scans. ${ }^{[10]}$ This approach is incorporated in the SA health policy. ${ }^{[7]}$ The observation that $85 \%$ of women in our study had an ultrasound scan implies that pregnant women in SA accept scans as a positive prenatal intervention.

Racial differences in the risk of birth defects have been observed. ${ }^{[11]}$ These may be related to genetic susceptibilities, dietary deficiencies and various exposures. A USA study found that African Americans were at a low risk of cardiac, genitourinary and craniofacial malformations, but at a higher risk of musculoskeletal defects compared with whites. ${ }^{[11]}$ As the majority of the women in the present study were black African (Table 1) and lethal central nervous system abnormalities were the most common, it is worthwhile to investigate the cause.

Folic acid supplementation has been shown to reduce the incidence of neural tube defects and subsequently fortification of staple foods has been implemented in SA to prevent folate deficiency. This has led to a notable decline in the occurrence of neural tube defects. ${ }^{[12]}$ According to the global Burden of Disease profile for SA, the contribution of congenital abnormalities to disease burden showed the largest decrease, falling by 32\% from 1990 to 2010. ${ }^{[13]}$ In contrast, it has been shown that the uptake of folic acid supplementation was low among women who carried fetuses with neural tube defects in sub-Saharan Africa. ${ }^{[14]}$ We did not assess compliance with regard to folic acid intake in the current study.

All women in our unit were counselled by a social worker prior to discharge. Several studies have shown that a termination decision is difficult and can be associated with stress and a feeling of ambivalence, and sometimes also clinical depression and anxiety ${ }^{[15]}$ Overall psychological morbidity among women who opted to terminate a pregnancy owing to an ultrasound-diagnosed anomaly has been shown to be significant and long-lasting. ${ }^{[15]}$

Our results support findings from a previous SA study which showed that $(i)$ respondents were generally accepting of feticide in the case of lethal fetal anomalies and (ii) even high doses of $\mathrm{KCl}$ are safe for this procedure. ${ }^{[3]}$ In addition, our study suggests that the current referral system is not efficient. Patients' delayed decisionmaking and subsequent investigations result in a long interval between a conclusive diagnosis and eventual feticide. We did not find any protocols or committees for guiding decision-making in the case of LTOP related to fetal anomalies in SA. Although such committees are involved in the process in high-income countries, their role is debated because of the associated delay in procedure. ${ }^{[9]}$

\section{Study limitations}

As this was a retrospective study, information may have been incomplete.

\section{Conclusion}

Our study has shown that the lack of a screening protocol as part of antenatal care contributes to late diagnosis of fetal anomalies, despite early booking for antenatal care. The referral interval from a primary to a tertiary centre was long, but in general less than 10 days. The use of $\mathrm{KCl}$ - alone or combined with lignocaine - was found to be safe for feticide.

We recommend that all pregnant women, regardless of their age, should have an ultrasound examination at their booking centre performed by appropriately trained staff. If this is not possible, patients should be referred to a tertiary centre in a timely manner. A standard protocol should be in place for antenatal screening of all pregnant women. We further also recommend that a national registry be established to keep a record of the number of late terminations performed related to fetal anomalies and the diagnoses that informed the decisions.

\section{Acknowledgements. None.}

Author contributions. FS and PSP designed the study and jointly wrote the manuscript. FS was responsible for statistical analysis and interpretation of the data. Funding. None.

Conflicts of interest. None.

\footnotetext{
1. Garne E, Loane M, Dolk H, et al. Prenatal diagnosis of severe structural congenital malformations in Europe. Ultrasound Obstet Gynecol 2005;25(1):6-11. https://doi.org/10.1002/uog.1784

2. Hamida EB, Ayadi I, Bezzine A, et al. Termination of pregnancy for fetal anomaly in a Tunisian population. S Afr J Obstet Gynaecol 2017;23(2):69-70. https://doi.org/10.7196/sajog.1159

3. Govender L, Moodley J. Late termination of pregnancy by intracardiac potassium chloride 3. Govender L, Moodley J. Late termination of pregnancy by intracardiac potassium chloride
injection: 5 years' experience at a tertiary referral centre. S Afr Med J 2012;103(1):47-51. https:// doi.org/10.7196/samj.6006

4. Moodley K. Feticide and late termination of pregnancy: Five levels of ethical conflict. Obstet Gynaecol Forum 2008;18(3):93-95. https://doi.org/10.4314/ogf.v18i3.30597
} 


\section{RESEARCH}

5. South Africa. Choice on Termination of Pregnancy Act No. 92 of 1996. https://www.parliament.gov za/storage/app/media/ProjectsAndEvents/womens_month_2015/docs/Act92of1996.pdf (accessed 1 June 2017).

6. Chervenak F, McCullough LB. Responsibly counselling women about the clinical management of pregnancies complicated by severe fetal anomalies. J Med Ethics 2012;38(7):397-398. https://dol org/10.1136/medethics-2012-100491

7. Department of Health (DoH). Guidelines for Maternity Care in South Africa. A manual for Clinics, Community Health Centres and District Hospitals. 4th ed. Pretoria: DoH, 2016:128-132.

8. Postoev VA, Grjibovski AM, Nieboer E, Odland JØ. Changes in detection of birth defects and perinatal mortality after introduction of prenatal ultrasound screening in the Kola Peninsula (North-West Russia): Combination of two birth registries. BMC Pregnancy Childbirth 2015;15:308. https://doi.org/10.1186/s12884-015-0747-1

9. Crane JP, LeFevre ML, Winborn RC, et al. A randomized trial of prenatal ultrasonographic crening: Impact on the detect

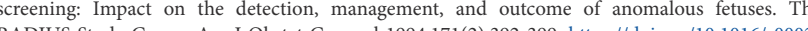
9378(94)70040-0

10. Hofmeyr GJ. Routine ultrasound examination in early pregnancy: Is it worthwhile in low-income countries? Ultrasound Obstet Gynecol 2009;34(4):367-370. https://doi.org/10.1002/uog.7352
11. Egbe AC. Birth defects in the newborn population: Race and ethnicity. Pediatr Neonatol 2015;56(3):183-188. https://doi.org/10.1016/j.pedneo.2014.10.002

12. Sayed A-R, Bourne D, Pattinson R, Nixon J, Henderson B. Decline in the prevalence of neural tube defects following folic acid fortification and its cost-benefit in South Africa. Birth Defects Res A Clin Mol Teratol 2008;82(4):211-216. https://doi.org/10.1002/bdra.20442

13. Institute for Health Metrics and Evaluation (IHME). Global Burden of Disease Study 2010. Globa Burden of Disease Profile: South Africa. Seattle: IHME, 2010. http://www.healthdata.org/sites/ default/files/files/country_profiles/GBD/ihme_gbd_country_report_south_africa.pdf (accessed June 2017)

14. Adeleye AO, Joel-Medewase VI. Awareness and uptake of measures for preventing CNS birth defects among mothers of affected children in a sub-Saharan African neurosurgeon's practice. Childs Nerv Syst 2015;31(12):2311-2317. https://doi.org/10.1007/s00381-015-2718-2

15. Salvesen KÅ, Øyen L, Schmidt N, Malt UF, Eik-Nes SH. Comparison of long-term psychological responses of women after pregnancy termination due to fetal anomalies and after perinatal los. Ultrasound Obstet Gynecol 1997;9(2):80-85. https://doi.org/10.1046/j.1469-0705.1997.09020080.x

Accepted 19 August 2019. 